\title{
A HIERARCHY OF LENGTH SCALES FOR WEAK SOLUTIONS OF THE THREE-DIMENSIONAL NAVIER-STOKES EQUATIONS*
}

\author{
J. D. GIBBON ${ }^{\dagger}$
}

\author{
To Dave Levermore on his 60 th birthday with friendship and appreciation
}

\begin{abstract}
Moments of the vorticity are used to define and estimate a hierarchy of time-averaged inverse length scales for weak solutions of the three-dimensional, incompressible Navier-Stokes equations on a periodic box. The estimate for the smallest of these inverse scales coincides with the inverse Kolmogorov length, but thereafter the exponents of the Reynolds number rise rapidly for correspondingly higher moments. The implications of these results for the computational resolution of small scale vortical structures are discussed.
\end{abstract}

Key words. Navier-Stokes, weak solutions, moments of vorticity, inverse length scales.

AMS subject classifications. 35Q30, 76D05.

\section{Introduction}

Resolution issues in computations of solutions of the three-dimensional NavierStokes equations are not only closely associated with the problem of regularity but they also raise the question of how resolution length scales can be defined and estimated. The Kolmogorov school of statistical turbulence suggests that a system of volume $L^{3}$ has a cut-off in its $-5 / 3$ energy spectrum at $k_{c}=\lambda_{k}^{-1} \sim L^{-1} R e^{3 / 4}$, which is known as the inverse Kolmogorov length. The wave-numbers $k>k_{c}$ are considered to lie in what is called the dissipation range $[1,2,3]$. Significant energy lying in this range can provoke intermittent events in the vorticity and strain fields characterized by violent, spiky departures away from space-time averages whose corresponding statistics appear to be non-Gaussian in character $[4,5,6,7,8]$, although intermittent events may also be associated with the inertial range $\left(k<k_{c}\right)$. Whether significant energy actually cascades down to the micro/nano-scales where the equation fails to be a valid model is intimately entwined not only with the open question of regularity but also with the role of the Navier-Stokes equations as a limit of kinetic theory $[9,10]$. This phenomenon continues to pose severe computational challenges $[11,12,13,14]$. In statistical physics the objects that are used to study intermittency are the ensembleaveraged velocity structure functions

$$
\left\langle|u(\boldsymbol{x}+\boldsymbol{r})-u(\boldsymbol{x})|^{p}\right\rangle_{\text {ens.av. }} \sim r^{\zeta_{p}}
$$

the departure of whose exponents $\zeta_{p}$ from linear ${ }^{1}$ is thought to be caused by inertial range intermittent behaviour $[1,2,3]$. It is clear, however, that these structure functions are not best suited for Navier-Stokes analysis; the task of this paper is to discuss what could replace these in the Navier-Stokes context and what information could be gleaned from them. While higher gradients of the velocity field would undoubtedly capture intermittent behaviour, they would be unreachable computationally for all practical purposes. A better diagnostic of spikiness in Navier-Stokes solutions is a

\footnotetext{
* Received: Devember 13, 2010; accepted (in revised version): May 6, 2011.

†Department of Mathematics, Imperial College London, SW7 2AZ, UK (j.d.gibbon@ic.ac.uk).

${ }^{1}$ Kolmogorov predicted a linear relation between $\zeta_{p}$ and $p$; departure from this is called 'anomalous scaling' and is usually manifest by $\zeta_{p}$ lying on a concave curve below linear for $p>3$ [1]. The two coincide for $p=3$.
} 
sequence of $L^{p}$-norms, or higher moments, of the vorticity $\boldsymbol{\omega}=\operatorname{curl} \boldsymbol{u}$, defined through the set of frequencies $(p=2 m$ for $m>1)$

$$
\Omega_{m}(t)=\left(L^{-3} \int_{\mathcal{V}}|\boldsymbol{\omega}|^{2 m} d V\right)^{1 / 2 m},
$$

where the domain $\mathcal{V}=[0, L]^{3}$ is taken to be periodic. The basic frequency associated with the domain is given by $\varpi_{0}=\nu L^{-2} . \Omega_{1}^{2}$ is the enstrophy per unit volume which is related to the energy dissipation rate, whereas the higher moments will naturally pick up events at smaller scales.

The setting is the incompressible $(\operatorname{div} \boldsymbol{u}=0)$, forced, three-dimensional NavierStokes equations for the velocity field $\boldsymbol{u}(\boldsymbol{x}, t)$ :

$$
\partial_{t} \boldsymbol{u}+\boldsymbol{u} \cdot \nabla \boldsymbol{u}=\nu \Delta \boldsymbol{u}-\nabla p+\boldsymbol{f}(\boldsymbol{x}) .
$$

Traditionally, most estimates in Navier-Stokes analysis have been found in terms of the Grashof number $G r$, which is expressed in terms of the root mean square $\left(f_{r m s}^{2}=L^{-3}\|\boldsymbol{f}\|_{2}^{2}\right)$ of the divergence-free forcing $\boldsymbol{f}(\boldsymbol{x})$ (see $[15,16,17,18]$ ), but it would be more helpful to express these in terms of the Reynolds number Re to facilitate comparison with the results of statistical physics. The definitions of $G r$ and $R e$ are

$$
G r=L^{3} f_{r m s} \nu^{-2}, \quad R e=U_{0} L \nu^{-1} .
$$

Doering and Foias [19] used the idea of defining $U_{0}$ as

$$
U_{0}^{2}=L^{-3}\left\langle\|\boldsymbol{u}\|_{2}^{2}\right\rangle_{T},
$$

where the time average $\langle\cdot\rangle_{T}$ over an interval $[0, T]$ is defined by

$$
\langle g(\cdot)\rangle_{T}=\underset{g(0)}{\limsup } \frac{1}{T} \int_{0}^{T} g(\tau) d \tau .
$$

Clearly, $G r$ is fixed provided $\boldsymbol{f}$ is $L^{2}$-bounded, while $R e$ is the system response to this forcing. A brief look at Leray's energy inequality,

$$
\frac{1}{2} \frac{d}{d t} \int_{\mathcal{V}}|\boldsymbol{u}|^{2} d V \leq-\nu \int_{\mathcal{V}}|\boldsymbol{\omega}|^{2} d V+\|\boldsymbol{f}\|_{2}\|\boldsymbol{u}\|_{2},
$$

shows why this definition of $U_{0}$ is of value - it leads to

$$
\left\langle\Omega_{1}^{2}\right\rangle_{T} \leq \varpi_{0}^{2} G r \operatorname{Re}+O\left(T^{-1}\right) .
$$

With some very mild technical restrictions on the forcing ${ }^{2}$, Doering and Foias [19] then showed that Navier-Stokes solutions obey $G r \leq c R e^{2}$. This turns (1.8) into

$$
\left\langle\Omega_{1}^{2}\right\rangle_{T} \leq c \varpi_{0}^{2} R e^{3}+O\left(T^{-1}\right) .
$$

In fact $\nu\left\langle\Omega_{1}^{2}\right\rangle_{T}$ is the time-averaged energy dissipation rate per unit volume over $[0, T]$ and allows us to form and bound from above the inverse Kolmogorov length scale $\lambda_{k}^{-1}$ :

$$
\lambda_{k}^{-4}=\frac{\nu\left\langle\Omega_{1}^{2}\right\rangle_{T}}{\nu^{3}} \quad \Rightarrow \quad L \lambda_{k}^{-1} \leq c R e^{3 / 4}+O\left(T^{-1 / 4}\right) .
$$

\footnotetext{
${ }^{2}$ Doering and Foias [19] took narrow-band forcing around a specific wave-number but a wavenumber spectrum which is cut off both above and below is sufficient.
} 
An estimate for the inverse Taylor micro-scale $\lambda_{\text {Tms }}^{-1}$ can also be found from (1.9):

$$
L \lambda_{T m s}^{-1}:=L\left(\frac{\left\langle\|\boldsymbol{\omega}\|_{2}^{2}\right\rangle_{T}}{\left\langle\|\boldsymbol{u}\|_{2}^{2}\right\rangle_{T}}\right)^{1 / 2} \leq c R e^{1 / 2}+O\left(T^{-1 / 2}\right) .
$$

Both these upper bounds gratifyingly coincide with the results of statistical turbulence theory $[1,2,3]$ although the fact that they are bounds allows for structures to occur in a flow whose natural scales are larger [20]. The question is now clear: can we construct and bound from above a sequence of inverse length scales associated with the higher moments $\Omega_{m}$ ?

\section{A scaling property and length scale estimates}

Leray's energy inequality (1.7) is valid for weak solutions and thus the estimate (1.9) is rigorous, although the existence and uniqueness of solutions for arbitrarily long times remain an open problem. While it is possible to subscribe to the view that difficulties in flow resolution could be a symptom of the lack of uniqueness of weak solutions, in tandem it ought also to be acknowledged that these difficulties may simply be caused by the practical challenges of working on a system where even the naturally largest scale (other than $L$ ) lies close to the limit of what can currently be resolved. The spirit of this paper is such that results on weak solutions are assumed to be sufficiently physical to reflect the reality of turbulent flows, provided $T$ is taken large enough ${ }^{3}$. This strategy allows the estimation of an infinite hierarchy of time averages of powers of the $\Omega_{m}$ for weak solutions on $[0, T]$ without having to appeal to point-wise estimates that the solution of the regularity problem would require. In turn, these time averages allow us to define and explore the natural length scales inherent in the system. The following result was proved in [21] under the assumption that strong solutions exist. Here it is demonstrated for weak solutions:

THEOREM 2.1. Weak solutions of the three dimensional Navier-Stokes equations satisfy

$$
\left\langle\left(\varpi_{0}^{-1} \Omega_{m}\right)^{\alpha_{m}}\right\rangle_{T} \leq c R e^{3}+O\left(T^{-1}\right), \quad 1 \leq m \leq \infty
$$

where $c$ is a uniform constant and

$$
\alpha_{m}=\frac{2 m}{4 m-3}
$$

REMARK 2.1. The exponent $\alpha_{m}=2 m /(4 m-3)$ within $(2.1)$ appears as a natural scaling of the Navier-Stokes equations, consistent with the application of Hölder and Sobolev inequalities. Note that when $m=1$ the value $\alpha_{1}=2$ is consistent with (1.9).

Proof. The proof is based on a result of Foias, Guillopé, and Temam [22] (their Theorem 3.1) for weak solutions which, when modified in the manner of Doering and Foias [19], furnishes us with the time averaged estimate

$$
\left\langle H_{N}^{\frac{1}{2 N-1}}\right\rangle_{T} \leq c_{N} L^{-1} \nu^{\frac{2}{2 N-1}} R e^{3}+O\left(T^{-1}\right),
$$

\footnotetext{
${ }^{3}$ While existence and uniqueness of solutions is easily proved for small values of $T[15,16,17]$, larger values than this are necessary to make sense of long-time averages.
} 
where

$$
H_{N}=\int_{\mathcal{V}}\left|\nabla^{N} \boldsymbol{u}\right|^{2} d V=\int_{\mathcal{V}_{k}} k^{2 N}|\hat{\boldsymbol{u}}|^{2} d^{3} k,
$$

and $H_{1}=\int_{\mathcal{V}}|\nabla \boldsymbol{u}|^{2} d V=\int_{\mathcal{V}}|\boldsymbol{\omega}|^{2} d V$. An interpolation between $\|\boldsymbol{\omega}\|_{2 m}$ and $\|\boldsymbol{\omega}\|_{2}$ is found using $H_{N}$

$$
\|\boldsymbol{\omega}\|_{2 m} \leq c_{N, m}\left\|\nabla^{N-1} \boldsymbol{\omega}\right\|_{2}^{a}\|\boldsymbol{\omega}\|_{2}^{1-a}, \quad a=\frac{3(m-1)}{2 m(N-1)},
$$

for $N \geq 3$. $\|\boldsymbol{\omega}\|_{2 m}$ is now raised to the power $\alpha_{m}$, which is to be determined, and the time average of this is estimated as

$$
\begin{aligned}
\left\langle\|\boldsymbol{\omega}\|_{2 m}^{\alpha_{m}}\right\rangle_{T} & \leq c_{N, m}^{\alpha_{m}}\left\langle\left\|\nabla^{N-1} \boldsymbol{\omega}\right\|_{2}^{a \alpha_{m}}\|\boldsymbol{\omega}\|_{2}^{(1-a) \alpha_{m}}\right\rangle_{T} \\
& =c_{N, m}^{\alpha_{m}}\left\langle\left(H_{N}^{\frac{1}{2 N-1}}\right)^{\frac{1}{2} a \alpha_{m}(2 N-1)} H_{1}^{\frac{1}{2}(1-a) \alpha_{m}}\right\rangle_{T} \\
& \leq c_{N, m}^{\alpha_{m}}\left\langle H_{N}^{\frac{1}{2 N-1}}\right\rangle_{T}^{\frac{1}{2} a \alpha_{m}(2 N-1)}\left\langle H^{\frac{(1-a) \alpha_{m}}{2-a \alpha_{m}(2 N-1)}}\right\rangle_{T}^{1-\frac{1}{2} a \alpha_{m}(2 N-1)}
\end{aligned}
$$

An explicit upper bound in terms of $R e$ is available only if the exponent of $H_{1}$ within the average is unity; that is

$$
\frac{(1-a) \alpha_{m}}{2-a \alpha_{m}(2 N-1)}=1
$$

This determines $\alpha_{m}$, uniformly in $N$, as

$$
\alpha_{m}=\frac{2 m}{4 m-3} .
$$

Using the estimate in (2.3), and that for $\left\langle H_{1}\right\rangle$, the result follows. The constant $c_{N, m}$ can be minimized by choosing $N=3 . c_{3, m}$ does not blow up even when $m=\infty$; thus we take the largest value of $c_{3, m}^{\alpha_{m}}$ and call this $c$.

\section{Definition of the inverse length scales}

Based on the definition of the inverse Kolmogorov length $\lambda_{k}^{-1}$ in (1.10) a generalization of this to a hierarchy of inverse lengths $\lambda_{m}^{-1}$ suggests the definition

$$
\left(L \lambda_{m}^{-1}\right)^{2 \alpha_{m}}:=\left\langle\left(\varpi_{0}^{-1} \Omega_{m}\right)^{\alpha_{m}}\right\rangle_{T} .
$$

The $\lambda_{m}$ are interpreted as resolution lengths in the space-time averaged sense for $1 \leq m \leq \infty$ :

$$
L \lambda_{m}^{-1} \leq c R e^{3 / 2 \alpha_{m}}+O\left(T^{-1 / 2 \alpha_{m}}\right) .
$$

Many turbulent structures have natural inverse gross length scales lying in the range between $R e^{1 / 2}$ and $R e^{3 / 4}$, but crinkles forming at finer scales may ultimately grow to be dominant and then become the cause of resolution difficulties $[1,2,3,13,14]$. For $m>1$ the $\lambda_{m}$ are interpreted here as the length scales corresponding to these deeper intermittent events. The upper bounds displayed in (3.2), as the Table shows, range 
from the inverse Kolmogorov length $R e^{3 / 4}$ at $m=1$ to $R e^{3}$ for $m=\infty$. Computationally it may be hard to get far beyond $m=1$ : for example, $m=9 / 8$ corresponds to $R e^{1}$, which is close to modern resolutions even for modest values of $R e$. Thereafter the rise in the exponent $3 / 2 \alpha_{m}$ is steep. Indeed, in the very high $m$ limit, the $R e^{3}$ bound has an exponent four times greater than the Kolmogorov length; this lies well below molecular scales where the equations are invalid. An interesting question is

\begin{tabular}{||c|c|c|c|c|c|c|c||}
\hline$m$ & 1 & $9 / 8$ & $3 / 2$ & 2 & 3 & $\cdots$ & $\infty$ \\
\hline $3 / 2 \alpha_{m}$ & $3 / 4$ & 1 & $3 / 2$ & $15 / 8$ & $9 / 4$ & $\cdots$ & 3 \\
\hline$d_{m}$ & 3 & 2 & 1 & $3 / 5$ & $1 / 2$ & $\cdots$ & 0 \\
\hline
\end{tabular}

TABLE 3.1. Values of the Re-exponent $3 / 2 \alpha_{m}=3\left(1-\frac{3}{4 m}\right)$, and $d_{m}=\frac{3}{4 m-3}$.

how the existence of this continuum of finer scales might be interpreted physically? To do so rigorously without a regularity proof is difficult, but a very informal physical interpretation is possible in terms of the familiar concept of a cascade. One of the simplest cascade models is the so-called $\beta$-model of Frisch, Sulem, and Nelkin [23] who, in analogy with Mandelbrot's ideas [24], modelled a Richardson cascade by taking a vortex of scale $\ell_{0} \equiv L$ and then allowed a cascade of daughter vortices, each of scale $\ell_{n}$. The idea was based on domain halving at each step such that $\ell_{0} / \ell_{n}=2^{n}$. The self-similarity dimension $d$ (similar to fractal dimension) was then introduced by considering the number of offspring at each step as $2^{d}: 2$ for the halving of a line; 4 for the halving of each direction in the plane; and likewise 8 for the cube. $d$ is then formally allowed to take non-integer values. In $d$ dimensions the corrections to the usual Kolmogorov scaling calculations for velocity, turn-over time etc appeared as multiplicative factors proportional to $\left(\ell_{0} / \ell_{n}\right)^{(3-d) / 3}$; see $[1,23]$. Equating the turnover and viscous times in the standard manner one arrives at $\left(\ell_{d}\right.$ is their viscous dissipation length $)^{4}$

$$
\ell_{0} / \ell_{d} \sim R e^{\frac{3}{d+1}}
$$

This gives the usual Kolmogorov inverse scale of $R e^{3 / 4}$ in a fully three-dimensional domain, but is shifted upwards for smaller values of $d$. Taking this idea as our physical analogy we compare (3.3) to the upper bound in (3.2) to get

$$
d_{m}+1=2 \alpha_{m} \quad \Rightarrow \quad d_{m}=\frac{3}{4 m-3},
$$

where an $m$-label has been appended to $d$. Thus we are able to assign a corresponding self-similarity dimension $d_{m}$ to lower-dimensional vortical structures that require values of $m>1$ for their resolution. Note that $d_{m}$ never goes negative. Models more sophisticated than the $\beta$-model, such as the bi-fractal and multi-fractal models [1], are more difficult to use as analogies as they would require data fitting.

\footnotetext{
${ }^{4}$ The integer $n$ labels the cascade: $m$ labels the higher moments as in (1.2), but does not necessarily take integer values. One choice is to take $n=m$, which means that each moment would correspond to a step in the cascade. In [23] $d$ is not specified but is illustrative of the calculation necessary when applying Kolmogorov's theory in non-integer dimensions.
} 


\section{REFERENCES}

[1] U. Frisch, Turbulence: The Legacy of A.N. Kolmogorov, Cambridge University Press, Cambridge, 1995.

2] P.A. Davidson, Turbulence, Oxford University Press, Oxford, 2004

[3] A. Tsinober, An Informal Conceptual Introduction to Turbulence, 2nd edition, Springer, 2009.

[4] G.K. Batchelor and A.A. Townsend, The nature of turbulent flow at large wave-numbers, Proc. R. Soc. Lond. A., 199, 238-255, 1949.

[5] A.Y.-S. Kuo and S. Corrsin, Experiments on internal intermittency and fine-structure distribution functions in fully turbulent fluid, J. Fluid Mech., 50, 285-320, 1971.

[6] C. Meneveau and K. Sreenivasan, The multi-fractal nature of turbulent energy dissipation, J. Fluid Mech., 224, 429-484, 1991.

[7] K. Sreenivasan, Fractals and multifractals in fluid turbulence, Ann. Rev. Fluid Mech., 23, 539-600, 1991.

[8] B.W. Zeff, D.D. Lanterman, R. McAllister, R. Roy, E.J. Kostelich, and D.P. Lathrop, Measuring intense rotation and dissipation in turbulent flows, Nature 421, 146-149, 2003.

[9] C. Bardos, F. Golse, and C.D. Levermore, Fluid dynamic limits of kinetic equations I. Formal derivations, J. Stat. Phys, 63, 323-344, 1991.

[10] C. Bardos, F. Golse, and C.D. Levermore, Fluid dynamic limits of kinetic equations II: Convergence proofs for the Boltzmann equation, Commun. Pure Appl. Math., 46, 667-753, 1993.

[11] U. Frisch and S. Orszag, Turbulence: Challenges for theory and experiment, Physics Today, January, 24-32, 1990.

[12] G.E. Karniadakis and S. Orszag, Nodes, modes and flow codes, Physics Today, March, 34-42, 1993.

[13] A. Vincent and M. Meneguzzi, The dynamics of vorticity tubes of homogeneous turbulence, J. Fluid Mech., 258, 245-254, 1994.

[14] J. Schumacher, B. Eckhardt, and C.R. Doering, Extreme vorticity growth in Navier-Stokes turbulence, Phys. Lett. A, 374, 861-864, 2010.

[15] P. Constantin and C. Foias, Navier-Stokes Equations, The University of Chicago Press, Chicago, 1988.

[16] R. Temam, Navier-Stokes Equations and Non-linear Functional Analysis, CBMS-NSF Regional Conference Series in Applied Mathematics, SIAM Press, Philadelphia, 1983.

[17] C. Foias, O. Manley, R. Rosa, and R. Temam, Navier-Stokes Equations and Turbulence, Cambridge University Press, Cambridge, 2001.

[18] C.R. Doering and J.D. Gibbon, Applied Analysis of the Navier-Stokes Equations, Cambridge University Press, Cambridge, 1995.

[19] C.R. Doering and C. Foias, Energy dissipation in body-forced turbulence, J. Fluid Mech., 467, 289-306, 2002.

[20] J.C. Vassilicos and J.C.R. Hunt, Fractal dimensions and spectra of interfaces with application to turbulence, Proc. R. Soc. Lond., A, 435, 505-534, 1991.

[21] J.D. Gibbon, Regularity and singularity in solutions of the three-dimensional Navier-Stokes equations, Proc. Royal Soc A, 466, 2587-2604, 2010.

[22] C. Foias, C. Guillopé, and R. Temam, New a priori estimates for Navier-Stokes equations in Dimension 3, Commun. Partial Diff. Equat., 6, 329-359, 1981.

[23] U. Frisch, P.-L. Sulem, and M. Nelkin, A simple dynamical model of intermittent fully developed turbulence, J. Fluid Mech., 87, 719-736, 1978.

[24] B. Mandelbrot, Intermittent turbulence in self-similar cascades: Divergence of high moments and dimension of the carrier, J. Fluid Mech., 62, 331-358, 1974. 